\title{
ESTUDO DE FLA VONÓDES E FENÓIS TOTAIS EM ESPÉCIES DE ALLAMANDA BLANCHETII, HIBISCUS ROSA SINESIS E MUSSAENDA ALICIA COLETADAS NA CIDADE DE FEIRA DE SANTANA
}

\author{
Jéssica Fernanda Ribeiro Oliveira'; ${ }^{1}$ Maria de Fátima Mendes Paixão² ; Heiddy \\ Márquez Alvarez ${ }^{3}$ e Lucas Souza da Silva ${ }^{4}$ \\ 1. Bolsista PROBIC/UEFS, Graduando em Licenciatura em Química, Universidade Estadual de Feira de Santana, e- \\ mail: jessicafernanda11@ @otmail.com \\ 2. Orientadora, Departamento de Ciências Exatas, Universidade Estadual de Feira de Santana, e-mail: \\ fpaixao100@gmail.com \\ 3. Co-Orientadora, Departamento de Ciências Exatas, Universidade Estadual de Feira de Santana, e-mail: \\ marquezheiddy@gmail.com \\ 4. Participante do LAPRON, Departamento de Saúde, Universidade Estadual de Feira de Santana, e-mail: \\ $\underline{\text { lsuefs@gmail.com }}$
}

PALAVRAS-CHAVE: Fenóis totais; flavonoides; pigmentos.

\section{INTRODUÇÃO}

As antocianinas são pigmentos vegetais, responsáveis por uma grande variedade de cores observadas em flores, frutos, algumas folhas, caules e raízes de plantas, que podem variar do vermelho vivo ao violeta/azul. (Castañeda, 2009). Quimicamente, esses pigmentos são compostos fenólicos pertencentes ao grupo dos flavonoides, que são importantes por atuarem na copigmentação das antocianinas, através de mecanismo de complexação intermolecular, tornando mais estável a molécula antociânica. (Filho \& Lima; 2011, 2002).

Segundo Navarro (2005), "a presença de flavonoides em espécies vegetais, parece estar relacionada com funções de defesa (proteção contra raios ultravioleta, fungos, e bactérias), e seus pigmentos servem de atração a agentes polinizadores."

De acordo com Nascimento (2009), na Mussaenda Alicia existe uma alta concentração de fenólicos na fração em acetato de etila, possivelmente relacionada com a presença de flavonóides, taninos e cumarinas detectadas nos testes químicos. Já as pétalas de Hibiscus rosa sinensis apresentam maior conteúdo de antocianinas $(\mathrm{p}<0,05)$, sendo considerada fonte de elevado teor do pigmento (teor superior a $200 \mathrm{mg} / 100 \mathrm{~g}$ ) (Teixeira et. al. 2008).

\section{MATERIAIS E MÉTODOS}

Inicialmente foi preparada uma solução metanólica de cloreto de alumínio a 5\%, em um balão volumétrico de $50 \mathrm{ml}$, adicionando $2,5 \mathrm{~g}$ de cloreto de alumínio e avolumando com metanol grau espectroscópico. Como a solução não ficou translúcida, foram feitas duas filtrações à vácuo. Em seguida, foram preparados os extratos das flores (com Etanol), que após filtrados e secos (rotoevaporação), foram usados para fazer três amostras com $0,01 \mathrm{~g}$ de extrato, preparadas em três béqueres (secos, tarados e identificados). Essa massa foi transferida para três balões volumétricos de $10 \mathrm{~mL}$ e avolumados com metanol grau espectroscópico. Dessa forma se obtêm soluções de concentração $1000 \mu / \mathrm{mL}$.

O conteúdo fenólico total foi determinado através do método espectrofotométrico de Folin-Ciocalteau $(\lambda=750)$, utilizando-se ácido gálico como padrão (PERES et al., 2009). O teor de flavonóides do extrato metanólico foi determinado também por 
espectrofotometria $(\lambda=425 \mathrm{~nm})$, utilizando-se cloreto de alumínio $\left(\mathrm{AlCl}_{3}\right)$ para possibilitar a quantificação e a quercetina como padrão interno (WOISKY, 1996; BANOV, 2006). Os extratos passaram por uma extração com Hexano (1:1) a fim de retirar os carotenos do extrato.

\section{RESULTADOS E/OU DISCUSSÃO (ou Análise e discussão dos resultados)}

\section{Determinação do conteúdo de Flavonoides Totais}

Em cada análise foram separados 11 tubos de ensaios e protegidos da luz com papel alumínio, etiquetados como 1A, 1B, 1C, 2A, 2B, 2C, 3A, 3B, 3C, B1 e B2. As soluções metanólicas (1,2 e 3) foram consideradas soluções mães para outro teste em triplicata. Nos tubos 1A, 1B e 1C foram adicionados $1500 \mu \mathrm{L}$ da solução mãe 1 , repetindo o procedimento com as outras soluções, a saber: $2 \mathrm{~A}, 2 \mathrm{~B}, 2 \mathrm{C}, 3 \mathrm{~A}, 3 \mathrm{~B}, 3 \mathrm{C}$. O preparo de B1 e B2 seguiu o mesmo procedimento, substituindo a amostra por metanol. Logo em seguida, adicionou-se em todos os tubos anteriormente citados $200 \mu \mathrm{L}$ da solução metanólica de $\mathrm{AlCl}_{3}(5 \%)$ e $3400 \mu \mathrm{L}$ da solução metanólica de Ácido Acético (5\%), homogeneizando em vortex e mantendo-se em repouso por 30 minutos. A leitura da absorbância foi feita em espectrofotômetro a $425 \mathrm{~nm}$. A determinação da concentração foi realizada através dos valores das absorbâncias obtidas, de acordo com a curva de calibração feita por Maira em 05/12/2016 através das absorbâncias de soluções padrão Quercitina $(10,20,30,40$ e $50 \mu \mathrm{g} / \mathrm{mL})$. A equação da reta de acordo com a curva de calibração é: $\mathrm{Abs}=0,021 \mathrm{C}-0,034 \mathrm{com} \mathrm{R}^{2}=0,997$.

\section{Determinação do conteúdo de Fenóis Totais}

Em cada análise foi separado 11 tubos de ensaios, protegidos da luz com papel alumínio e etiquetados como 1A, 1B, 1C, 2A, 2B, 2C, 3A, 3B, 3C, B1 e B2. As soluções metanólicas (1,2 e 3 ) foram consideradas soluções mães para mais um teste em triplicata. Nos tubos 1A, 1B e 1C foram adicionados $100 \mu \mathrm{L}$ da solução mãe 1 , o mesmo ocorreu com as outras soluções. Logo em seguida, adicionou-se $1000 \mu \mathrm{L}$ de água ultrapura e $200 \mu \mathrm{L}$ do reagente Folin Ciocalteu, foi homogeneizado em vortex e mantido em repouso por 30 minutos. A leitura da absorbância foi feita em espectrofotômetro a $750 \mathrm{~nm}$. A determinação da concentração foi realizada através dos valores das absorbâncias obtidas, de acordo com a curva de calibração feita por Maira em 05/12/2016 através das absorbâncias de soluções padrão Ácido Gálico (50, 100, 150, 200, 250, 300 e $350 \mu \mathrm{g} / \mathrm{mL}$ ). A equação da reta de acordo com a curva de calibração é: $\mathrm{Abs}=0,002 \mathrm{C}+0,011$ com $\mathrm{R}^{2}=0,992$.

O teor de fenólicos e flavonoides é consideravelmente maior na espécie Allamanda Blachetti. Na Mussaenda Alicia foi necessário realizar partição liquido-líquido com hexano (1:1), essa partição foi feita usando $50 \mathrm{~mL}$ de extrato etanólico de cada espécie usando 5 porções de $10 \mathrm{~mL}$ de hexano para evitar a formação de fungos, para remover esteroides, terpenos, acetofenonas e carotenoides do extrato etanólico bruto.

Os flavonoides por sua vez assim como alguns fenóis apresentam duas características cruciais, a primeira se refere a complexarem com as antocianinas dando uma maior estabilidade as mesmas, atuando como co-pigmentos. A segunda grande característica se refere a sua atuação como antioxidantes, conhecidos como capturadores de radicais livres. Foi possível determinar a concentração de fenólicos e flavonoides em $\mu \mathrm{g} / \mathrm{mL}$, nas três espécies estudadas (Allamanda Blanchetii, Hibiscus rosa sinensis e Mussaenda Alicia). Os resultados obtidos são apresentados na Tabela 1, onde podemos perceber 
que a Allamanda apresenta uma elevada concentração desses componentes (flavonoides e fenóis totais).

Tabela 1. Comparação de Fenólicos e Flavonóides nas 3 espécies.

\begin{tabular}{|c|c|c|c|c|c|c|c|c|c|}
\hline & $\mathrm{H} 1$ & $\mathrm{H} 2$ & $\mathrm{H} 3$ & $\mathrm{AB} 1$ & $\mathrm{AB} 2$ & $\mathrm{AB} 3$ & $\begin{array}{c}\text { MA } \\
1 *\end{array}$ & $\begin{array}{c}\text { MA } \\
2^{*}\end{array}$ & $\begin{array}{c}\text { MA } \\
3^{*}\end{array}$ \\
\hline $\begin{array}{c}\text { Concentração } \\
\text { de Fenólicos } \\
(\mu \mathrm{g} / \mathrm{mL})\end{array}$ & 36,17 & 53,17 & 45.33 & 88,16 & 94,5 & 123,5 & 52,8 & 46,7 & 49,1 \\
\hline $\begin{array}{c}\text { Concentração } \\
\text { de } \\
\begin{array}{c}\text { Flavonóides } \\
(\mu \mathrm{g} / \mathrm{mL})\end{array}\end{array}$ & 11,98 & 13,95 & 11,31 & 19 & 20,6 & 24,31 & 12,87 & 10,54 & 12,14 \\
\hline $\begin{array}{c}\text { Concentração } \\
\text { dos Extratos } \\
\text { Metanólicos } \\
(\mu \mathrm{g} / \mathrm{mL})\end{array}$ & 1320 & 1640 & 1180 & 1200 & 1580 & 1490 & 1380 & 1210 & 1330 \\
\hline
\end{tabular}

Legenda: H (Hibiscus rosa sinesis), AB (Allamanda Blanchetii) e MA (Mussaenda Alicia). 1 (Amostra 1), 2 (Amostra 2) e 3 (Amostra 3). * Hexano.

\section{CONSIDERAÇÕES FINAIS (ou Conclusão)}

O estudo realizado nos mostrou que Allamanda Blanchetii é a espécie que possui maior teor de fenóis totais e de flavonoides. A resposta dada pelas outras duas espécies (Hibiscus rosa sinesis e Mussaenda Alicia) não foi significativa para os objetivos pleiteados.

\section{REFERÊNCIAS}

BANOV, D. et al. Caracterização do extrato seco de Ginkgo biloba L.em formulações de uso tópico. Acta Farm. Bonaerense 25 (2): 219-24. 2006.

Dissertação Mestrado em Fármacos e Medicamentos - Faculdade de Ciências Farmacêuticas, Universidade de São Paulo, São Paulo, 1996.

FILHO, A. B. D. M. et. al. Química de Alimentos. Produção alimentícia. Rede e-Tec Brasil. UFRPE/CODAI, 2011.

KELEBEK H; CANBAS A; SELLI S; SAUCIER C; JOUDES M \& GLORIES Y. (2006) Influence of different maceration times on theanthocyanins composition of wines made from Vitis vinifera L. cvs. Bogazkere and Öküzgözü. Journal of Food Engineering, 77: 1012-1017. 
LIMA, V. L. A. G. D. et. al. Polpa Congelada De Acerola: Efeito Da Temperatura Sobre Os Teores De Antocianinas E Flavonóis Totais. Rev. Bras. Frutic, Jaboticabal - SP, v. 24, n. 3, p. 669-670, Dezembro 2002.

NASCIMENTO, R. F. D. Perfil Fitoquímico e Concentração de Fenólicos Totais na Espécie Mussaenda alicia Hort. 32a Reunião Anual da Sociedade Brasileira de Química (SBQ), 2009.

NAVARRO, D. D. F. Estudo Químico, biológico e farmacológico das espécies Allamanda blanchetti e Allamanda schottii Pohl para obtenção de frações e moléculas bioativas de potencial terapêutico. Tese de Doutorado. Universidade Federal de Santa Catarina (UFSC), Florianópolis, 2005.

PERES, M. T. L. P et. al. Estudos químicos e biológicos de Microgramma vacciniifolia (Lansd. \& Fisch.) Copel (Polypodiaceae). Química Nova, v. 32, n. 4, p. 897-901.2009.

WOISKY. R.G. Método de controle químico de amostras de própolis. 74f. 